\title{
Mortalidad de Pacientes Oncológicos vinculados a Neutropenia
} \section{Mortality of Oncological Patients linked to Neutropenia}

*Correspondencia: drzambrano2013@yahoo.com Teléfono [593] 02281744

Conflicto de intereses: Los autores declaran no tener conflictos de intereses.

Fondos: Ver la página 23

Recibido: 1 Enero 2018 Aceptado: 11 Agosto 2018 Publicado: 30 Abril 2019

Membrete bibliográfico: García C, Vivar J, Frías-Toral E, Zambrano C. Mortalidad de Pacientes Oncológicos vinculados a Neutropenia. Rev. Oncol. Ecu 2019;29(1):12-26.

ISSN: 2661-6653

DOI: https://doi.org/10.33821/279

Copyright García, et al. Este artículo es distribuido bajo los términos de Creative Commons Attribution License, el cual permite el uso y redistribución citando la fuente y al autor original.

\section{Carlos García Cruz'1,2 Dosefina Vivar Maldonado1, Evelin Frías-Toral1 Cecilio Zambrano García2*(iD)}

1. Servicio de Cuidados Intensivos, Solca-Guayaquil.

2. Universidad de Especialidades "Espíritu Santo" (UESS). Samborondón, Ecuador, Departamento de Postgrados.

Introducción: La neutropenia absoluta se presenta comúnmente en pacientes oncológicos en tratamiento activo de quimioterapia, lo que determina aumento de morbi-mortalidad y en muchos casos el manejo clínico debe realizarse en una Unidad de Cuidados Intensivos (UCI). El objetivo del estudio es reportar la mortalidad y supervivencia de un grupo de pacientes neutropénicos ingresados en una UCI.

Métodos: En el área de UCI del Instituto Oncológico Nacional "Dr. Juan Tanca Marengo", SolcaGuayaquil, se realizó una investigación observacional retrospectiva, del período Enero 2015 a diciembre 2016. Se seleccionaron pacientes mayores de 18 años con recuentos de neutrófilos $\leq 500$ células $/ \mathrm{mm}^{3}$. Las variables fueron sexo, tipo de cáncer, diagnóstico de ingreso, SOFA, ventilación mecánica, falla Hepática, falla renal, tipo de Diagnóstico Infeccioso, tipos de muestras recabadas de cultivos con resultados positivos, germen aislado y mortalidad.Se utiliza análisis descriptivo, de supervivencia, Regresión de COX y análisis de Kaplan Meier.

Resultados: Ingresaron al estudio 99 casos, 50 mujeres (51 \%), la edad media de $33.7 \pm 24$ años. Los diagnósticos oncológicos más prevalentes fueron Leucemia Linfoblástica Aguda 39 casos (39.4 \%) y Leucemia Mieloide Aguda 11 casos (11.1\%). La mortalidad fue de 58 casos (58.6 \%) con una supervivencia de $19.7 \pm 4.8$ días. La Regresión de COX (OR) para hombres fue de 0.7 (IC $950.43-$ 1.246) $P=0.25$. La supervivencia por edad fue mayor en el grupo de 10 a 29 años (37.4 \pm 19.8 días), en el grupo de 50 a 59 años, fue de $4.2 \pm 1.1$ días $(P<0.05)$. No existieron diferencias estadísticas de la supervivencia según el motivo de internación.

Conclusión: En el presente estudio la mortalidad reportada en pacientes con neutropenia ingresados a UCl fue del (58.6\%). La supervivencia no está asociada al sexo y la mejor supervivencia está asociada con la menor edad. No existieron diferencias estadísticas de la supervivencia según el motivo de internación. 
Palabras Claves: NEUTROPENIA, NEUTROPENIA FEBRIL INDUCIDA POR QUIMIOTERAPIA, MORTALIDAD, GRUPOS DE EDAD

DOI: $10.33821 / 279$

\section{Abstract}

Introduction: Absolute neutropenia is commonly seen in cancer patients undergoing active chemotherapy treatment, which determines increased morbidity and mortality and in many cases clinical management must be performed in an Intensive Care Unit (ICU). The objective of the study is to report the mortality and survival of a group of neutropenic patients admitted to an ICU.

Methods: In the ICU area of the National Oncological Institute "Dr. Juan Tanca Marengo ", SolcaGuayaquil, a retrospective observational research was conducted, from January 2015 to December 2016. Patients older than 18 years were selected with neutrophil counts $\leq 500$ cells / mm3. The variables were sex, cancer type, diagnosis of admission, SOFA, mechanical ventilation, Hepatic failure, renal failure, type of Infectious Diagnosis, types of samples collected from cultures with positive results, isolated germ and mortality. Survival, COX regression and Kaplan Meier analysis were used.

Results: 99 cases, 50 women (51\%), the mean age of $33.7 \pm 24$ years entered the study. The most prevalent oncological diagnoses were Acute Lymphoblastic Leukemia 39 cases (39.4\%) and Acute Myeloid Leukemia 11 cases (11.1\%). Mortality was 58 cases (58.6\%) with a survival of $19.7 \pm 4.8$ days. The COX (OR) Regression for men was 0.7 (IC $950.43-1.246) \mathrm{P}=0.25$. The survival by age was higher in the group of 10 to 29 years ( $37.4 \pm 19.8$ days), in the group of 50 to 59 years, it was $4.2 \pm 1.1$ days $(\mathrm{P}<0.05)$. There were no statistical differences in survival according to the reason for hospitalization.

Conclusion: In the present study, the mortality reported in patients with neutropenia admitted to the ICU was (58.6\%). Survival is not associated with sex and the best survival is associated with younger age. There were no statistical differences in survival according to the reason for hospitalization.

Keywords: NEUTROPENIA, CHEMOTHERAPY-INDUCED FEBRILE NEUTROPENIA, MORTALITY, AGE GROUPS

DOI: $10.33821 / 279$

\section{Introducción}

Los pacientes oncológicos tienen múltiples factores de riesgo para adquirir infecciones, llevando su condición a niveles críticos y alta mortalidad, lo que condiciona una selectividad con el fin de evitar el ensañamiento terapéutico para la admisión en la Unidad de Cuidados Intensivos ( $\mathrm{UCl}$ ). La neutropenia post quimioterapia es uno de los factores más relevantes en estos pacientes.

La neutropenia se define como un recuento absoluto de neutrófilos $<500$ células $/ \mathrm{mm}^{3}$, o $<1.000$ células $/ \mathrm{mm}^{3}$ con una disminución predecible a $<500$ células $/ \mathrm{mm}^{3}$ dentro de las próximas 48 horas del ingreso [1]. Existe una relación inversa entre el número de neutrófilos circulantes y el riesgo de infección, todos los pacientes con un recuento $<100$ células $/ \mathrm{mm}^{3}$ por más de 3 semanas desarrollan infecciones, generando que sean pacientes de alto riesgo [2]. 
Las causas de la neutropenia no solo se deben al efecto quimio y radioterápico también los síndromes mielodisplásicos y las malignidades hematológicas típicamente causan pancitopenia. Una minoría de los casos presenta neutropenia aislada. En las últimas décadas, el aumento de la intensidad del tratamiento en pacientes con cáncer se ha traducido en una mejor supervivencia [3], sin embargo esto ha contribuido a un mayor aumento de casos de neutropenia.

El "punto más bajo" en el recuento de neutrófilos y una neutropenia prolongada (Ej.: recuento de neutrófilos de $<500$ células $/ \mathrm{mm}^{3}$ por $>5$ días) son factores de riesgo mayores para el desarrollo de infecciones. Además de los cambios cuantitativos en el recuento de neutrófilos, las anormalidades en la función fagocítica u otras deficiencias en la respuesta inmune pueden aumentar significativamente el riesgo de infección en un huésped neutropénico. El momento del Nadir llega usualmente unos 10 días después del tratamiento, aunque esto puede variar dependiendo del agente quimioterapéutico administrado. La preocupación durante esta etapa es que en este momento la primera línea de defensa del cuerpo contra las infecciones está bajo y el paciente es más susceptible a infecciones y hemorragias. La dosis siguiente de quimioterapia solo se administra después de que los recuentos sanguíneos se hayan superado a un nivel seguro [4].

El riesgo de complicaciones graves depende principalmente de la duración de la neutropenia (> 7 días), presencia de comorbilidades, como la disfunción hepática o renal [6], la edad >60 años [5]. Sin embargo también hay reportes controversiales en los que el uso de ventilación mecánica, la presencia de falla hepática y la presencia de falla cardiaca predecían en un 41 \% la mortalidad con ninguna asociación de la neutropenia como factor de riesgo de mortalidad [6].

Dentro de las principales situaciones clínicas encontradas en pacientes neutropénicos críticamente enfermos, las infecciones gastrointestinales fueron las más frecuentes en un $31 \%$ seguidas de las respiratorias en $30 \%$ [7]. El tratamiento del cáncer ha contribuido a un número creciente de pacientes inmunocomprometidos con una mayor incidencia de infecciones nosocomiales; La inmunosupresión puede resultar en un mayor uso de antibióticos y más infecciones asociadas con microorganismos multirresistentes [8].

Los gérmenes más aislados en pacientes Neutropénicos con cáncer en las infecciones Hematológicas; son los organismos Gram Positivos, entre ellos: Estafilococo CoagulasaNegativo, Estreptococo del grupo Viridans, Estafilococo Aureus, Escherichia coli y Pseudomona Aeruginosa [9].

Generalmente la fuente de infección es más difícil de identificar en los Pacientes Neutropénicos (PN) que en los pacientes con función inmune normal, ya que los síntomas de la infección a menudo disminuyen. El espectro de patógenos potenciales es amplio y el diagnóstico precoz es esencial para guiar el tratamiento y minimizar la terapia farmacológica no esencial, el tratamiento antibiótico / anti fúngico empírico en las infecciones sospechosas debe adaptarse a cada paciente para maximizar las posibilidades de que la terapia sea microbiológicamente apropiada, En todos los PN, la terapia antibacteriana empírica de amplio espectro debe iniciarse inmediatamente después de que se hayan obtenido los hemocultivos y antes de que se hayan completado otras investigaciones [10]. Las infecciones poli microbianas se han triplicado en los últimos años 
siendo responsables del 25 al 30\% del total de infecciones bacterianas, y al menos el $80 \%$ tienen un germen Gram Negativo, enfatizándose la necesidad de la cobertura de amplio espectro en el tratamiento empírico de PN [11].

Para predecir adecuadamente la mortalidad con buena discriminación durante las primeras 24 horas de estancia en $\mathrm{UCl}$ de pacientes oncológicos críticos, se emplean los parámetros definidos por The Sequential Organ Failure Assessment (SOFA), y The Simplified Acute Physiology Score II (SAPS II), por sus siglas en inglés, respectivamente $[12,13]$. Reportes indican que durante los primeros días de ingreso a la UCI, el SOFA es un buen indicador tanto en las puntuaciones medias como altas, también aclara que si existiera un aumento del puntaje de SOFA a las 48 horas, independientemente del puntaje del ingreso, predice una tasa de mortalidad de al menos un 50 \% de los pacientes [14]. En un estudio se demostró que los pacientes con cánceres hematológicos con complicaciones mortales ingresados en la UCI tenían un riesgo de muerte a largo plazo y el marcador predictor SOFA es uno de los más fidedignos, al mismo tiempo que podría ayudar a evitar acciones fútiles con pacientes con SOFA mayor de 15 [15].

El objetivo del presente estudio es describir la mortalidad en pacientes oncológicos ingresados con neutropenia en una Unidad de terapia Intensiva.

\section{Materiales y Métodos}

En el área de Unidad de cuidados intensivos del Instituto Oncológico Nacional "Dr. Juan Tanca Marengo", Solca-Guayaquil, se realizó una investigación observacional retrospectiva de las historias clínicas del servicio del período Enero del 2015 a diciembre del 2016. El universo estuvo conformado de todos los pacientes que ingresaron al servicio con neutropenia, se seleccionaron los pacientes con neutropenia absoluta mayores de 18 años, con recuento de neutrófilos menor o igual a 500 células $/ \mathrm{mm}^{3}$, que tuvieron todos los datos analizables. El muestreo fue no probabilístico de tipo intencional. Una segunda fuente fueron los resultados de laboratorio, y la tercera fuente los informes anatomopatológicos.

Las variables documentadas fueron sexo, tipo de cáncer clasificado según tumores sólidos y Hematológicos, diagnóstico de ingreso a la Terapia Intensiva, SOFA al ingreso, ventilación mecánica, falla Hepática, falla renal, tipo de Diagnóstico Infeccioso según el CDC (siglas en inglés de Centers for Disease Control and Prevention), tipos de muestras recabadas de cultivos con resultados positivos, germen aislado y mortalidad.

Se excluyeron a pacientes que solicitaron el alta, pacientes con neutropenias absolutas postquimioterapia que no se pudo establecer la etapa o estadio del cáncer al momento del ingreso.

Se utiliza análisis descriptivo con frecuencias y porcentajes. Se utiliza el análisis de supervivencia, Regresión de COX y análisis de Kaplan Meier. El paquete estadístico utilizado fue SPSS 22.0 para PC. La significación estadística se estableció cuando el valor de $P<0.05$. 


\section{Resultados}

Ingresaron al estudio 99 casos, 50 (51\%) fueron mujeres y 49 (49\%) hombres, la edad media del grupo fue de $33.7 \pm 24$ años. No hubo diferencia de distribución ni de edad entre hombres y mujeres (Tabla 1), el grupo etáreo más prevalente fue el de $\geq 80$ años 41 casos ( $41.4 \%$ ), de 50 a 59 años 18 casos (18.2 \%) y de 40 a 49 años 12 casos (12.1\%), de 18 a 29 años 10 casos $(10.1 \%)$. Los diagnósticos oncológicos más prevalentes fueron Leucemia Linfoblástica aguda 39 casos (39.4\%) y Leucemia Mieloide aguda 11 casos (11.1\%) (Tabla 2).

La mortalidad reportada del grupo de pacientes con neutropenia ingresados a UCl fue de 58 casos (58.6\%). El $50 \%$ de pacientes fallecieron con diagnóstico de insuficiencia respiratoria 29 casos. El análisis de supervivencia estimó un promedio $19.7 \pm 4.8$ días en los pacientes que desarrollan neutropenia. La supervivencia no está asociada al sexo (Figura 1), ya que la Regresión de COX (OR) para hombres fue de 0.7 (IC $950.43-1.246$ ) P= 0.251 .

Tabla 1. Edad por sexo en el grupo de estudio.

\begin{tabular}{|l|c|c|c|}
\hline & $\begin{array}{c}\text { Mujer } \mathbf{n = 5 0} \\
\mathbf{( 5 1} \%)\end{array}$ & $\begin{array}{c}\text { Hombre } \mathbf{n = 4 9} \\
\mathbf{( 4 9} \%)\end{array}$ & $\boldsymbol{P}$ \\
\hline Edad & $36.8 \pm 26$ & $30.6 \pm 22$ & 0.206 \\
\hline
\end{tabular}

Figura 1. Función de supervivencia de acuerdo al Sexo

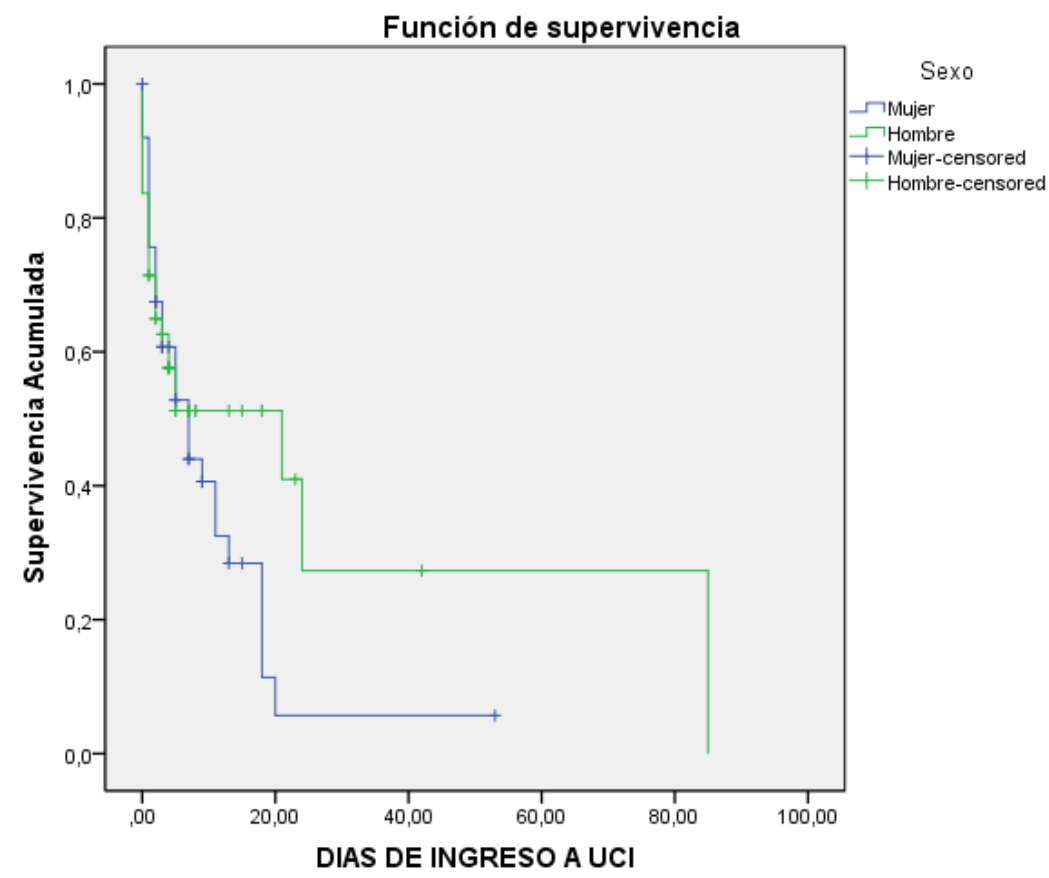


Tabla 2. Diagnósticos Oncológicos más prevalentes en pacientes con neutropenia ingresados a UCI.

\begin{tabular}{|l|c|c|c|}
\hline \multicolumn{1}{|c|}{ Diagnóstico Oncológico } & Mujer n=50 & Hombre=49 & Total =99 \\
\hline Leucemia linfoblástica aguda & $20(40 \%)$ & $19(38.8 \%)$ & $39(39.4 \%)$ \\
\hline Leucemia mieloide aguda & $6(12 \%)$ & $5(10.2 \%)$ & $11(11.1 \%)$ \\
\hline Leucemia linfoide & 0 & $6(12.2 \%)$ & $6(6.1 \%)$ \\
\hline Linfoma no Hodgkin difuso & $2(4 \%)$ & $4(8.2 \%)$ & $6(6.1 \%)$ \\
\hline Linfoma no Hodgkin folicular [nodular] & $2(4 \%)$ & $2(4.1 \%)$ & $4(4 \%)$ \\
\hline Tumor maligno del cuello del útero & $3(6 \%)$ & 0 & $3(3 \%)$ \\
\hline Enfermedad linfoproliferativa T-gamma & $2(4 \%)$ & 0 & $2(2 \%)$ \\
\hline Leucemia mieloide crónica & 0 & $2(4.1 \%)$ & $2(2 \%)$ \\
\hline Linfoma de células T, periférico y cutáneo & 0 & $2(4.1 \%)$ & $2(2 \%)$ \\
\hline Neumonía debida a 4 microorganismos infecciosos & $2(4 \%)$ & 0 & $2(2 \%)$ \\
\hline Tumor maligno del estómago & 0 & $2(4.1 \%)$ & $2(2 \%)$ \\
\hline Enfermedad linfoproliferativa SAl & $1(2 \%)$ & 0 & $1(1 \%)$ \\
\hline Enf. de las cuerdas vocales y de la laringe & 0 & $1(2 \%)$ & $1(1 \%)$ \\
\hline Hepatoblastoma & 0 & $1(2 \%)$ & $1(1 \%)$ \\
\hline Histiocitoma fibroso, maligno & $1(2 \%)$ & 0 & $1(1 \%)$ \\
\hline Insuficiencia respiratoria aguda & $1(2 \%)$ & 0 & $1(1 \%)$ \\
\hline Linfomas No Hodgkin & $1(2 \%)$ & 0 & $1(1 \%)$ \\
\hline Meduloblastoma melanotico & $1(2 \%)$ & 0 & $1(1 \%)$ \\
\hline Mieloma múltiple & $1(2 \%)$ & 0 & $1(1 \%)$ \\
\hline Rabdosarcoma & 0 & $1(2 \%)$ & $1(1 \%)$ \\
\hline Síndromes mielodisplásicos & $1(2 \%)$ & 0 & $1(1 \%)$ \\
\hline Tumor de células germinales, no seminomatoso & 0 & $1(2 \%)$ & $1(1 \%)$ \\
\hline Tumor maligno de la glándula tiroides & $1(2 \%)$ & 0 & $1(1 \%)$ \\
\hline Tumor maligno de la laringe & 0 & $1(2 \%)$ & $1(1 \%)$ \\
\hline Tumor maligno de la mama & $1(2 \%)$ & 0 & $1(1 \%)$ \\
\hline Tumor maligno de los huesos, cartílagos articulares & 0 & $1(2 \%)$ & $1(1 \%)$ \\
\hline Tumor maligno del colon & $1(2 \%)$ & 0 & $1(1 \%)$ \\
\hline Tumor maligno del endometrio & $1(2 \%)$ & 0 & $1(1 \%)$ \\
\hline Tumor maligno del lóbulo frontal & 0 & $1(2 \%)$ & $1(1 \%)$ \\
\hline Tumor maligno del peritoneo y del retroperitoneo & $1(2 \%)$ & 0 & $1(1 \%)$ \\
\hline Tumor maligno del seno maxilar & $1(2 \%)$ & 0 & $1(1 \%)$ \\
\hline
\end{tabular}

La supervivencia por edad fue mayor en el grupo de 10 a 29 años (37.4 \pm 19.8 días), la peor supervivencia se presentó en el grupo de 50 a 59 años, la cual fue de $4.2 \pm 1.1$ días. Estas diferencias de supervivencia son estadísticamente significativas $(P<0.05)$ (Tabla 3 y 4). La mejor supervivencia se observó en el grupo acumulado de 18 a 49 años versus el grupo de 50 años o más (Figura 2). 
Tabla 3. Supervivencia en días, de los pacientes neutropénicos ingresados a UCI.

\begin{tabular}{|c|c|c|c|c|}
\hline \multirow{3}{*}{ Edad* categoría } & \multicolumn{4}{|c|}{ Media } \\
\hline & \multirow[b]{2}{*}{ Estimación (Días) } & \multirow{2}{*}{$\begin{array}{c}\text { Error } \\
\text { estándar }\end{array}$} & \multicolumn{2}{|c|}{ Intervalo de confianza 95\% } \\
\hline & & & $\begin{array}{l}\text { Límite } \\
\text { inferior }\end{array}$ & $\begin{array}{l}\text { Límite } \\
\text { superior }\end{array}$ \\
\hline 18 a 29 años & 37.4 & 19.8 & 0 & 76.1 \\
\hline 30 a 39 años & 4.3 & 1.2 & 2.0 & 6.6 \\
\hline 40 a 49 años & 19.0 & 5.0 & 9.2 & 29.0 \\
\hline 50 a 59 años & 4.2 & 1.1 & 2.0 & 6.5 \\
\hline 60 a 69 años & 20.9 & 9.9 & 1.5 & 40.2 \\
\hline 70 a 79 años & 10.5 & 6.4 & 0 & 23.1 \\
\hline$>80$ años & 9.6 & 1.4 & 6.8 & 12.3 \\
\hline Todos & 19.8 & 4.8 & 10.4 & 29.1 \\
\hline
\end{tabular}

Chi $<$ Cuadrado= Log Rank (mantel-Cox) $=12.962, \mathrm{P}=0.044$.

Tabla 4. Resumen del procesamiento de casos por edad.

\begin{tabular}{|l|c|c|c|c|}
\hline & & & \multicolumn{2}{|c|}{ Censurados } \\
\cline { 3 - 5 } Edad* categoría & Total N & N de Eventos & N & Porcentaje \\
\hline 18 a 29 años & 10 & 5 & 5 & $50.0 \%$ \\
\hline 30 a 39 años & 7 & 3 & 4 & $57.1 \%$ \\
\hline $\mathbf{4 0}$ a 49 años & 12 & 7 & 5 & $41.7 \%$ \\
\hline $\mathbf{5 0}$ a 59 años & 18 & 15 & 3 & $16.7 \%$ \\
\hline 60 a 69 años & 7 & 4 & 3 & $42.9 \%$ \\
\hline 70 a 79 años & 4 & 3 & 1 & $25.0 \%$ \\
\hline >80 años & 41 & 21 & 20 & $48.8 \%$ \\
\hline Todos & 99 & 58 & 41 & $41.4 \%$ \\
\hline
\end{tabular}

No existieron diferencias estadísticas de la supervivencia según el motivo de internación (datos no mostrados).

El grupo de edad de los fallecidos fue de 51 a 70 años que representaron más de un $90 \%$ de mortalidad siendo el grupo de mayor prevalencia, el diagnóstico que fue representativo incluyo a los pacientes con choque séptico al ingreso tuvieron una mayor mortalidad en relación al 50 \% de pacientes que fallecieron con insuficiencia respiratoria (Tabla 4), los días de estancia en $\mathrm{UCl}$ de los PN fallecidos tuvieron un promedio de estancia en UCl entre 5 a 10 días mayor en $11 \%$ que con los pacientes que estuvieron 1 a 4 días, de acuerdo a la condición de ventilación el total de PN ingresados en Terapia Intensiva que estuvieron en ventilación mecánica, 88 \% de ellos fallecieron (51 casos), 29 casos atribuibles directamente a la insuficiencia respiratoria fallecieron (50\%), atribuibles a Falla Hepática y Falla Renal (Tabla 4). Los pacientes con más alto SOFA $>13$ tuvieron una alta mortalidad 16/16 casos con SOFA de 13 -14 y de 11/11 casos con SOFA de 15-24 (Tabla 5). Las infecciones más prevalentes en los pacientes fallecidos fueron la neumonía 40 casos (70\%), coexistieron las infecciones por catéter y enterocolitis (Tabla 6). La etiología más prevalente fue por Escherichia Coli Blee (+) 5 casos (14.7\%), Klebsiella Pneumoniae Kpc (+) 4 casos $(11.8 \%)$, (Tabla 7). 
Figura 2. Supervivencia por edad en pacientes neutropénicos ingresados en $\mathrm{UCl}$ con patología oncológica

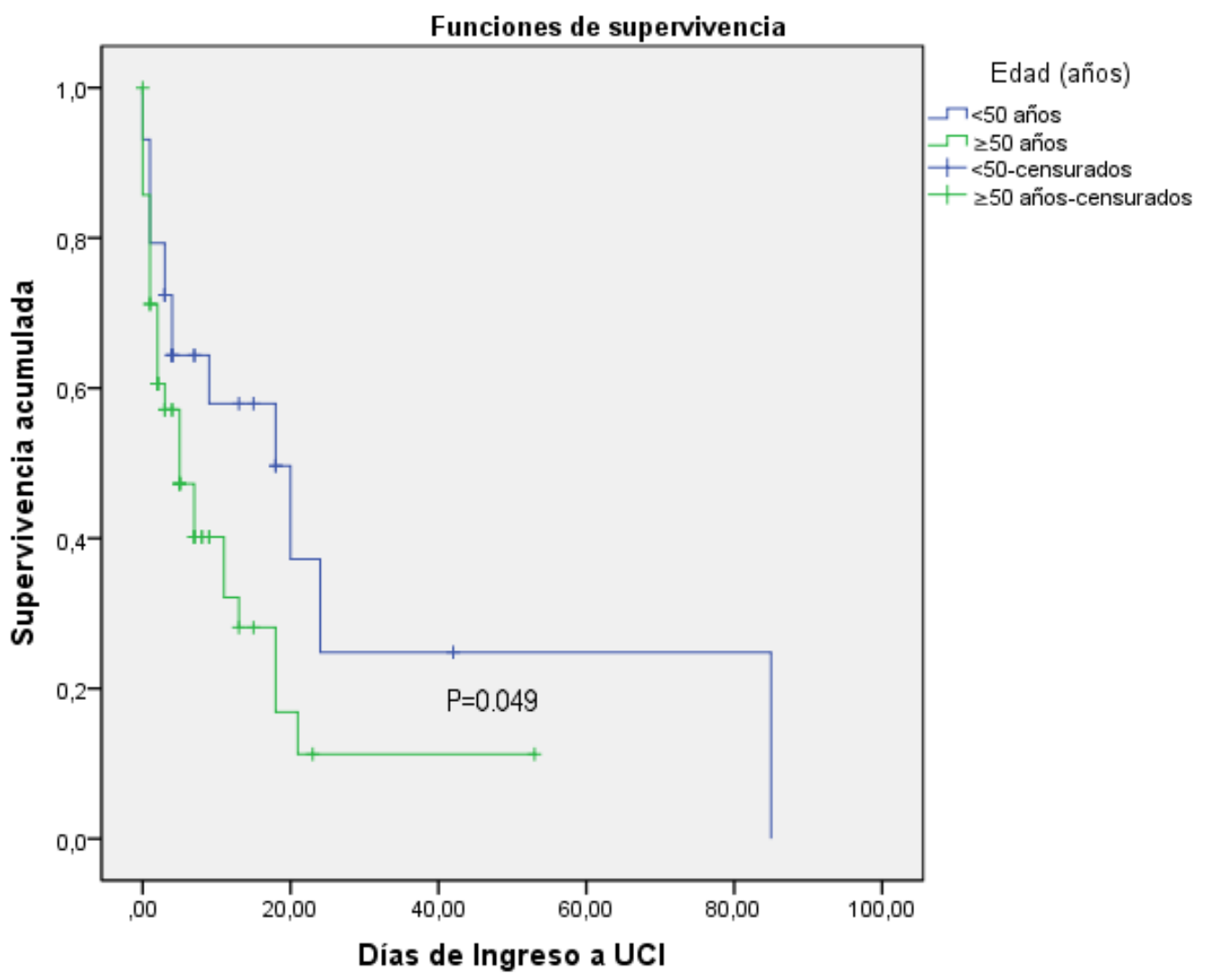

Tabla 4. Causa atribuible de muerte en los pacientes incluidos en el estudio.

\begin{tabular}{|l|c|c|}
\hline \multicolumn{1}{|c|}{ Fallecidos $\mathbf{n = 5 8}$} & Total $\mathbf{~ N}$ & Porcentajes \\
\hline Fallecidos con tratamiento de Ventilación Mecánica & 51 & $88.0 \%$ \\
\hline Fallecidos por insuficiencia respiratoria & 29 & $50.0 \%$ \\
\hline Fallecidos por Falla Hepática & 24 & $41.4 \%$ \\
\hline Fallecidos por Falla Renal & 25 & $43.1 \%$ \\
\hline
\end{tabular}

Tabla 5. SOFA (Acute Organ System Failure) en los pacientes fallecidos con neutropenia

\begin{tabular}{|l|c|c|c|}
\hline \multicolumn{1}{|c|}{ SOFA } & N=99 & Fallecidos $\mathbf{N = 5 8}$ & Porcentajes de mortalidad \\
\hline $\mathbf{0}$ a $\mathbf{6}$ & 14 & 0 & $0 \%$ \\
\hline $\mathbf{7}$ a $\mathbf{9}$ & 28 & 9 & $33.0 \%$ \\
\hline $\mathbf{1 0}$ a $\mathbf{1 2}$ & 30 & 22 & $73.0 \%$ \\
\hline $\mathbf{1 3}$ a $\mathbf{1 4}$ & 16 & 16 & $100.0 \%$ \\
\hline $\mathbf{1 5}$ a $\mathbf{2 4}$ & 11 & 11 & $100.0 \%$ \\
\hline
\end{tabular}


Tabla 6. Infecciones coexistentes y concomitantes en los pacientes fallecidos

\begin{tabular}{|l|c|c|}
\hline \multicolumn{1}{|c|}{ Diagnóstico } & $\mathbf{N = 5 8}$ & Porcentajes \\
\hline Neumonía & 40 & $70.0 \%$ \\
\hline Infección por catéter & 38 & $65.5 \%$ \\
\hline Enterocolitis & 30 & $51.7 \%$ \\
\hline Infección oral/Mucositis & 29 & $50.0 \%$ \\
\hline Infección de partes blandas & 29 & $50.0 \%$ \\
\hline Infección Urinaria & 18 & $31.0 \%$ \\
\hline Neumonía asociada a VM & 11 & $19.0 \%$ \\
\hline
\end{tabular}

Tabla 7. Etiologías de infecciones

\begin{tabular}{|l|c|c|}
\hline Gérmenes & $\begin{array}{c}\text { Total } \\
\mathbf{N}=34\end{array}$ & Porcentajes \\
\hline Escherichia Coli Blee (+) & 5 & $14.7 \%$ \\
\hline Klebsiella Pneumoniae Kpc (+) & 4 & $11.8 \%$ \\
\hline Klebsiella Pneumoniae & 4 & $11.8 \%$ \\
\hline Pseudomona Aeruginosa & 3 & $8.8 \%$ \\
\hline Escherichia Coli & 3 & $8.8 \%$ \\
\hline Burkholderia (P.) Cepacia & 3 & $8.8 \%$ \\
\hline Enterobacter Amnigenus & 3 & $8.8 \%$ \\
\hline Klebisella Ozaenae & 2 & $5.9 \%$ \\
\hline Klebsiella Pneumoniae Blee(+) & 2 & $5.9 \%$ \\
\hline Staphylococcus Aureus & 2 & $5.9 \%$ \\
\hline Enterobacter Aerogenes & 1 & $2.9 \%$ \\
\hline Serratia Fonticola & 1 & $2.9 \%$ \\
\hline Stenotrophomonas Maltophilia & 1 & $2.9 \%$ \\
\hline
\end{tabular}

\section{Discusión}

La UCl es esencial para el cuidado de los pacientes con cáncer. En estudios multicéntricos recientes, los pacientes con cáncer que ingresaron a Unidades de Cuidados Intensivos representaron hasta el $21 \%$ de todas las admisiones [16], además en la última década los avances tecnológicos y científicos han permitido mejores resultados en pacientes con cáncer que requieren admisión en terapia intensiva $[17,18]$. Se ha descrito que los PN ingresados en la $\mathrm{UCl}$ representan un $66 \%$ de mortalidad en estos pacientes al igual que los no neutropénicos [19].

El presente estudio la mortalidad fue del $58.6 \%$, al respecto un estudio que relacionó la mortalidad de los PN, se observó que las mujeres fallecieron un $8 \%$ más que los hombres [19]; sin embargo, en contraste con lo citado, el presente estudio no reporta diferencias de 
mortalidad por sexo, lo que se puede deducir que existen otros factores influyen en los resultados.

Un estudio realizado en el 2006 determinó que la edad era un factor de riesgo importante de mortalidad en los pacientes con cáncer ingresados en la terapia intensiva con una media de mayor o igual de 60 años [5]; no obstante, en el presente trabajo, los pacientes con una patología oncológica de base, que desarrollaron neutropenia y fallecieron, el grupo de edad de 50 años o más presentaron más de un $74 \%$ de mortalidad siendo el más representativo.

En el estudio de Supervivencia retrospectivo de 11 años de Legrand de pacientes con cáncer en estado crítico con neutropenia en sepsis y choque séptico se observó que la media de edad de pacientes fallecidos fue de 54 años [20], en cambio en este estudio el promedio de edad fue de $33.7 \pm 24$ años con diferencias claras del número de participantes.

Los pacientes que ingresan en la $\mathrm{UCl}$ con diagnóstico de choque séptico tienen una mortalidad de $63 \%$ [21], en contraste con los pacientes a los que a esa patología se agrega neutropenia y que presentan una mayor mortalidad $(80 \%)$ según lo recabado, pudiendo asumir que la condición de neutropenia en estos pacientes podría agravar la mortalidad en un $20 \%$. Así también, el 50 \% de pacientes fallecieron con diagnóstico de insuficiencia respiratoria, estos mismos resultados los visualizó Blot en su estudio retrospectivo de 4 años de neutropénicos ingresados en la Terapia Intensiva, donde se buscaba establecer los factores de pronósticos en mortalidad en relación a neoplasias hematológicas o tumores sólidos y las fallas orgánicas agudas confirmándose su alto impacto en la mortalidad [22].

En el presente estudio, los fallecidos tuvieron un rango de estancia en UCl entre 5 a 10 días similar a lo que encontró Regazzoni, donde la media fue de 6.6 días en pacientes neutropénicos fallecidos por choque séptico [23].

Los resultados encontrados en el estudio se asocian al Puntaje SOFA a partir de los 13 puntos, la mortalidad acumulada es del $100 \%$, resultados similares a estudios publicados [20]. La ventilación mecánica constituye un factor de riesgo ya que el $88 \%$ de los pacientes que fallecen fueron tratados de esta forma, comparativamente con el $78 \%$ de los pacientes que no fallecieron. Al igual que lo menciona un estudio, se considera a la ventilación mecánica un factor de riesgo en el pronóstico de los pacientes al igual que su impacto de un $41 \%$ en la mortalidad [24]. Se considera que la ventilación mecánica en Neutropénicos influye en un $53 \%$ en la mortalidad [6].

Al igual que la ventilación mecánica, la falla hepática al momento del ingreso representa otro factor de riesgo en la mortalidad de pacientes oncológicos críticamente enfermos con neutropenia; sin embargo, los artículos investigados proponen un rango de mortalidad entre el 40 y $60 \%$ [23-25]; mientras que, en el estudio realizado representa más de un $90 \%$ en la mortalidad del total de pacientes ingresados.

Del total de pacientes ingresados con diagnósticos infecciosos de: Infección por Catéter, Neumonía asociada a Ventilación Mecánica e Infección de foco Urinario presentaron un $100 \%$ en la mortalidad seguidos por $70 \%$ de los pacientes que fallecieron por Neumonía, los porcentajes similares entre las Mucositis, Enterocolitis e Infección de partes Blandas del 
$50 \%$ de los pacientes, lo que podría asociarse que los dispositivos invasivos y la ventilación mecánica en pacientes neutropénicos podría aumentarse como factores de riesgo de la mortalidad en estos pacientes, ya está descrito que la VM representa un 53\%de mortalidad, al igual que los pacientes con falla respiratoria aguda también se asociada a esta problemática $[6,13,26]$.

De la serie en estudio los que tuvieron resultados positivos en cultivos de punta de catéter, urocultivo fallecieron el $100 \%$ mientras que los pacientes con aspirado traqueal y hemocultivos fallecieron 80 y $79 \%$ respectivamente, según lo establecido en la literatura los pacientes con hemocultivos positivos fue una condicionante para documentar el proceso infeccioso y el germen causal pero en nuestra casuística indica que a pesar de ser alta lo recabado reportan que su mortalidad solo representan un $79 \%[1,10]$.

En la década pasada los gérmenes causales de infecciones en PN eran los gérmenes Gram Positivo, luego de un giro en los sitios de infecciones y asociado a nuevos factores de riesgo de estos pacientes los gérmenes Gram Negativos son los organismos más frecuentes de infecciones nosocomiales en las Terapias Intensivas, [9, 11]. El estudio demostró que los gérmenes más comunes causantes de infecciones en los $\mathrm{PN}$ fallecidos son los gérmenes Gram Negativos se encuentran en mayor proporción y en mayor porcentaje de impacto en la mortalidad de los pacientes neutropénicos, La klebsiella Pneumoniae tanto BLEE como KPC tiene un alta proporción en prevalencia en el estudio, sin embargo agente causales como Stafilococos Aureus también incidió con un $100 \%$ de mortalidad como resultado.

Nuevos estudios comparativos deberán realizarse en pacientes con y sin neutropenia con el objetivo de establecer diferencias que permitan discriminar el peso del efecto de la inmunosupresión en la mortalidad. Se recomienda realizar estudios de mayor temporalidad para generar una muestra más representativa. Adicionalmente, emplear un antibiótico de terapia empírica en pacientes neutropénicos con choque séptico para gérmenes Gram Negativos ya que son los más comunes en la incidencia de la mortalidad en este tipo de pacientes.

\section{Conclusiones}

En el presente estudio la mortalidad reportada en pacientes con neutropenia ingresados a $\mathrm{UCl}$ fue del $58.6 \%$, con una supervivencia de $19.7 \pm 4.8$ días. La supervivencia no está asociada al sexo y la mejor supervivencia está asociada con la menor edad. No existieron diferencias estadísticas de la supervivencia según el motivo de internación. 


\section{Agradecimientos}

Reconocemos a las personas que participaron indirectamente en el estudio tales como el personal técnico, pacientes y personal de la Unidad de Cuidados Intensivos del Instituto Oncológico Nacional "Dr. Juan Tanca Marengo", Solca-Guayaquil.

\section{Información adicional}

\section{Nota del Editor}

La Revista Oncología Ecu permanece neutral con respecto a los reclamos jurisdiccionales en mapas publicados y afiliaciones institucionales.

\section{Abreviaturas}

PN: Pacientes Neutropénicos.

UCI: Unidad de Cuidados Intensivos.

VM: Ventilación Mecánica.

SOFA: Acute Organ System Failure.

\section{Archivos Adicionales}

Ninguno declarado por los autores.

Fondos

Los fondos de la investigación fueron propios de los autores del presente artículo.

\section{Disponibilidad de datos y materiales}

Existe la disponibilidad de datos bajo solicitud al autor de correspondencia. No se reportan otros materiales.

Contribuciones de los autores

CGC, EFT, CZG realizaron la idea de investigación, revisión bibliográfica. JVM CGC, recolección de datos, escritura del artículo. EFT realizó el análisis crítico del artículo. CZG realizó las correcciones editoriales. Todos los autores leyeron y aprobaron la versión final del artículo.

Aprobación de ética y consentimiento para participar

No aplica a este estudio. 


\section{Consentimiento para publicación}

No aplica.

\section{Información de los autores}

Carlos García Cruz, Médico Intensivista del Instituto Oncológico Nacional "Dr. Juan Tanca Marengo", Solca-Guayaquil. Docente de Post grado Universidad de Especialidades Espíritu Santo (UEES). Medicina Crítica y Terapia Intensiva.

口@ carlos.garcia.cruz@gmail.com ( (593)4 2109041 - (593) 42109042 - 593 993284745.

Evelyn Frías Toral, Magíster en Investigación Clínica y Epidemiológica, Diplomado Nutrición Enteral y Parenteral. Coordinadora del Comité de Investigación del Instituto Oncológico Nacional "Dr. Juan Tanca Marengo", Solca-Guayaquil.

¿@evelynft@gmail.com

Josefina Vivar M, Especialista en Terapia Intensiva, tratante del Instituto Oncológico Nacional “Dr. Juan Tanca Marengo”, Solca-Guayaquil.

@ pepitavivar@hotmail.com 1593981383941

Cecilio Zambrano, Cirujano General, tratante del Instituto Oncológico Nacional "Dr. Juan Tanca Marengo", Solca-Guayaquil.

drzambrano2013@yahoo.com. 593989973150

미 (iD) https://orcid.org/0000-0003-1851-2445

Revisiones por pares

Acceda a la revisión de pares académicos en el siguiente enlace: https://publons.com/review/4516232/

\section{Referencias}

Abreviaturas en la referencias DOI: Digital Object Identifier PMID: PubMed Identifier SU: Short URL
1. Wisplinghoff $\mathrm{H}$, Seifert H, Wenzel RP, Edmond MB. Current trends in the epidemiology of nosocomial bloodstream infections in patients with hematological malignancies and solid neoplasms in hospitals in the United States. Clin Infect Dis. 2003;36(9):1103-10. DOI: 10.1086/374339

2. Hughes W, Armstrong D, Bodey GP, Bow EJ, Brown AE, Calandra T, et al. 2002 Guidelines for the Use of Antimicrobial Agents in Neutropenic Patients with Cancer, Clinical Infectious Diseases 2002;34(6):730751. DOI: $10.1086 / 339215$ 
3. Talcott JA, Finberg R, Mayer RJ, Goldman L. The Medical Course of Cancer Patients With Fever and Neutropenia: Clinical Identification of a Low-Risk Subgroup at Presentation. Arch Intern Med.1988;148(12):2561-2568. DOI:10.1001/archinte.1988.00380120031007

4. Brenner H, Gondos A, Arndt V. Recent Major Progress in Long-Term Cancer Patient Survival Disclosed by Modeled Period Analysis. Journal of Clinical Oncology 2007;25(22):3274-3280. DOI: 10.1200/JC0.2007.11.3431

5. Flowers CR, Seidenfeld J, Bow EJ, Karten C, Gleason C, Hawley DK, et al. Antimicrobial prophylaxis and outpatient management of fever and neutropenia in adults treated for malignancy: American Society of Clinical Oncology clinical practice guideline. J Clin Oncol. 2013;31(6):794-810. DOI: $10.1200 / \mathrm{JCO} .2012 .45 .8661$

6. Kress JP, Christenson J, Pohlman AS, Linkin DR, Hall JB. Outcomes of critically ill cancer patients in a university hospital setting. Am J Respir Crit Care Med. 1999;160(6):1957-61. DOI: 10.1164/ajrccm.160.6.9812055

7. Rabagliati R, Fuentes G, Orellana E, Oporto J, Domínguez I, Benítez R, et al. Etiología de episodios de neutropenia febril en pacientes adultos con cáncer hematológico y de órganos sólidos en el Hospital Clínico Universidad Católica, Santiago-Chile. Rev. Chil. Infectol. 2009;26(2):106-113. DOI: $\underline{10.4067 / S 0716-10182009000200001}$

8. Vincent JL, Sakr Y, Sprung CL, Ranieri VM, Reinhart K, Gerlach H, et al. Sepsis Occurrence in Acutely III Patients Investigators. Sepsis in European intensive care units: results of the SOAP study. Crit Care Med. 2006;34(2):344-53. DOI: 10.1097/01.CCM.0000194725.48928.3ª

9. Hospital de SOLCA. Secretaria General. Estadística de los Registro de SOLCA. Guayaquil: Secretaría General del Hospital de SOLCA; 2012- 2014.

10. Freifeld AG, Bow EJ, Sepkowitz KA, Boeckh MJ, Ito JI, Mullen CA, et al. Clinical practice guideline for the use of antimicrobial agents in neutropenic patients with cancer: 2010 update by the infectious diseases society of america. Clin Infect Dis. 2011;52(4):e56-93. DOI: 10.1093/cid/cir073.

11. Mateos MC, Arguiñano JM, Ardaiz MA, Oyarzábal FJ. Infecciones en pacientes oncohematológicos no trasplantados. Anales Sis San Navarra 2005;28(1):59-81. SU: bit.ly/2r4npna

12. Vincent J, Moreno R, Takala J, Willatts S, De Mendonça A, Bruining H, et al. The SOFA (Sepsis-related Organ Failure) to describe organ dysfunction/failure. Intensive Care Med 1996;22:707-710. SU: bit.ly/2W2HDAB

13. Guiguet M, Blot F, Escudier B, Antoun S, Leclercq B, Nitenberg G. Severity-of-illness scores for neutropenic cancer patients in an intensive care unit: Which is the best predictor? Do multiple assessment times improve the predictive value? Crit Care Med. 1998;26(3):488-93. PMID: 9504577

14. Ferreira FL, Bota DP, Bross A, Mélot C, Vincent JL. Serial evaluation of the SOFA score to predict outcome in critically ill patients. JAMA. 2001;286(14):1754-8. PMID: 11594901.

15. Cornet AD, Issa Al, van de Loosdrecht AA, Ossenkoppele GJ, Strack van Schijndel RJ, Groeneveld AB. Sequential organ failure predicts mortality of patients with a haematological malignancy needing intensive care. Eur J Haematol.2005;74(6):511-6. DOI: 10.1111/j.1600-0609.2005.00418.x

16. Taccone FS, Artigas AA, Sprung CL, Moreno R, Sakr Y, Vincent JL. Characteristics and outcomes of cancer patients in European ICUs. Crit Care. 2009;13(1):R15. DOI: 10.1186/cc7713

17. Staudinger T, Stoiser B, Müllner M, Locker GJ, Laczika K, Knapp S, et al. Outcome and prognostic factors in critically ill cancer patients admitted to the intensive care unit. Crit Care Med. 2000;28(5):1322-8. PMID: 10834673

18. Soares M, Salluh JIF, Torres VBL, Leal JVR, Spector N. Short- and long-term outcomes of critically ill patients with cancer and prolonged ICU length of stay. Chest. 2008;134(3):520-526. DOI: $10.1378 /$ chest.08-0359. 
19. Souza-Dantas VC, Salluh J, Soares M. Impact of neutropenia on the outcomes of critically ill patients with cancer: a matched case-control study, Annals of Oncology 2011;22(9):2094-2100. DOI: 10.1093/annonc/mdq711

20. Legrand M, Max A, Peigne V, Mariotte $E$, Canet $E$, Debrumetz A, et al. Survival in neutropenic patients with severe sepsis or septic shock. Crit Care Med. 2012;40(1):43-9. DOI: 10.1097/CCM.0b013e31822b50c2.

21. Quenot JP, Binquet C, Kara F, Martinet O, Ganster F, Navellou JC, et al. The epidemiology of septic shock in French intensive care units: the prospective multicenter cohort EPISS study. Crit Care 2013;17(2):R65. DOI: $10.1186 / \operatorname{cc} 12598$

22. Blot F, Guiguet M, Nitenberg G, Leclercq B, Gachot B, Escudier B. Prognostic factors for neutropenic patients in an intensive care unit: respective roles of underlying malignancies and acute organ failures. Eur J Cancer. 1997;33(7):1031-7. PMID: 9376183.

23. Penack $O$, Becker $C$, Buchheidt $D$, Christopeit $M$, Kiehl $M$, von Lilienfeld-Toal $M$, et al. Management of sepsis in neutropenic patients: 2014 updated guidelines from the Infectious Diseases Working Party of the German Society of Hematology and Medical Oncology (AGIHO). Ann Hematol. 2014;93(7):1083-95. DOI: $10.1007 / \mathrm{s} 00277-014-2086-0$.

24. Mokart D, Darmon M, Resche-Rigon M, Lemiale V, Pène F, Mayaux J, et al. Prognosis of neutropenic patients admitted to the intensive care unit. Intensive Care Med 2015;41(2):296-303. DOI: 10.1007/s00134-014-3615-y.

25. Pène $F$, Percheron $S$, Lemiale $V$, Viallon $V$, Claessens $Y E$, Marqué $S$, et al. Temporal changes in management and outcome of septic shock in patients with malignancies in the intensive care unit. Crit Care Med 2008;36(3):690-6. DOI: 10.1097/CCM.0B013E318165314B.

26. Vandijck DM, Benoit DD, Depuydt PO, Offner FC, Blot SI, Van Tilborgh AK, et al. Impact of recent intravenous chemotherapy on outcome in severe sepsis and septic shock patients with hematological malignancies. Intensive Care Med. 2008;34(5):847-55. DOI:10.1007/s00134-008-1002-2. 